\title{
EDITORIAL
}

For reprint orders, please contact: reprints@futuremedicine.com

\section{Melanoma treatment guided by a panel of miRNA biomarkers}

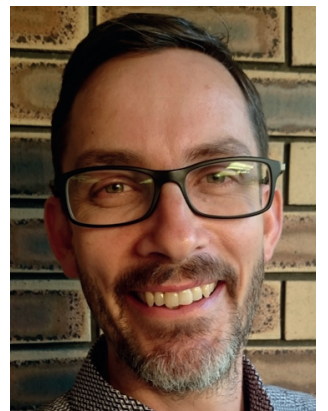

Mitchell S Stark* “...the melanoma-related seven miRNA was found to characterize overall survival of melanoma patients better than both serum lactate dehydrogenase and S100B...”
First draft submitted: 12 March 2017; Accepted for publication: 28 March 2017; Published online: 10 May 2017
Melanoma incidence is continuing to rise in Australia [1], US [2] and in the UK and Europe [3]. Metastatic patients have a poor prognosis and are expensive to treat whereas if melanoma is caught in its in situ stage (or in precursor lesions), the 5-year survival is $>99 \%$. In melanoma, primary prevention strategies and early detection of primary lesions offer the best outcome but rely heavily on self-monitoring and the expertise of medical professionals. After surgical excision of the lesion, patients are asked to return to their treating clinician at regular intervals which spans from months to years, dependent upon the American Joint Committee on Cancer tumor stage (I-IV) [4]. If the lesion has invaded beyond the epidermis, a sentinel lymph node is commonly biopsied, which is important for accurate staging of the patient to devise clinical follow-up and treatment regimens. In most cases the sentinel lymph node has no detectable cells, therefore, the patient is staged as I or II depending on the level of invasion. In patients with stage II tumors, their relative 5-year survival rates range from approximately $80 \%$ (stage IIA) to
$50 \%$ (stage IIC) [4]. These differences in survival strongly indicate that in a large proportion of patients, metastatic spread has occurred and was not detected by conventional methods or the patient was lost to follow-up. It is evident that these practices are not absolute and disease can be missed, reoccur or manifest as metastatic disease without the observation of a primary or palpable lesion. In clinical practice, there is currently a lack of reliable, sensitive and specific predictive biomarkers for detecting early melanoma progression. Considering the complexities of offering a diagnosis of the degree of spread beyond the primary site, having the ability to rapidly identify predictors of melanoma progression in patients would therefore be a significant clinical tool.

Potential markers to detect melanoma progression have been investigated for many years with varying levels of success. Serum lactate dehydrogenase (LDH) levels have been reported to be elevated in melanoma patients but the performance of this marker reduces as disease progresses [5-8]. Due to these limitations, it is not routinely used in
*Dermatology Research Centre, The University of Queensland Diamantina Institute at the Translational Research Institute, Brisbane, Queensland, Australia; m.stark@uq.edu.au

\section{KEYWORDS:}

- biomarker • imaging • melanoma

- miRNA • monitoring • serum • skin

- stage $\bullet$ tissue 


\section{"Future melanoma follow-up and treatment regimens should consider the utility of miRNAs as a companion diagnostic and prognostic aid in a clinical setting."}

all centers. To address this clinical unmet need, we recently identified a panel of highly specific and sensitive miRNA biomarkers that have the potential to monitor melanoma progression in both tissue and serum in a clinical setting [9]. In this study, we first showed that a panel of 'melanoma-related' miRNAs (MELmiR-17) were able to predict the stage, recurrence and survival of patients when its expression was measured in melanoma tissues derived from metastases (stages III and IV) [9]. Currently, we are assessing if this biomarker panel could be used as a companion diagnostic tool to assist in histopathological diagnosis of ambiguous early melanocytic lesions. Additionally, we found that in a minimally invasive blood test, a sevenmiRNA panel (MELmiR-7) derived from the 'MELmiR-17' panel, detected the presence of melanoma (relative to no melanoma controls) with high sensitivity (93\%) and specificity ( $\geq 82 \%)$ [9]. Importantly, in stage IV patients, members of the MELmiR-7 panel were able to detect an increase in tumor burden in $100 \%$ of cases [9]. One particular miRNA from the panel, miR-211, has long been associated as a melanocyte/melanoma related miRNA [10]. In a conditional inference tree analysis, we showed that stage at blood draw together with miR-211 expression could be used to triage patients based on overall survival (OS) status [9]. Specifically, upon diagnosis with stage IV disease and prior to therapy, miR-211 expression was able to discern survival based on high (median survival = 4.8 months, CI: 4.5-5.9) and low expression (median survival $=2.7$ years, CI: 1.7-NA) [9]. Taken together, the MELmiR-7 panel was found to characterize OS of melanoma patients better than both serum LDH and S100B (delta-log likelihood = 11; $\mathrm{p}<0.001$ ) [9].

The accurate staging of individuals is of the utmost importance to devise appropriate follow-up and treatment regimens. We found that the circulating miRNA expression of our panel was able to discern between disease-free controls and stage IV disease with a near perfect area under the curve (AUC; AUC = 0.99) [9]. Moreover, members of the panel could discriminate between stages with an AUC for stages I/II versus IV and stages III versus IV of 0.99 respectively [9]. With further development, this panel has the potential to be able to upstage patients without observable disease. This may mean they could qualify for the current targeted therapies. It has been well documented in the literature that patients with a lower disease burden, treated with first-line therapy, exhibit an improvement in OS [11-13]. However, generally speaking, patients present to their oncologist with varying degrees of metastatic spread. It is therefore evident that a more precise way to monitor disease burden and to predict treatment response is required to complement and improve current practices.

Until relatively recently, metastatic melanoma patients had no viable treatment options for disseminated melanoma. With the advent of targeted- (BRAF and MEK inhibition) and immuno-therapy (anti-PD1/PDL1 and anti-CTLA4 antibodies), we are witnessing improvements in progression-free survival. However, in a fair proportion of patients, there has not been a dramatic improvement in patient's OS, with many experiencing a relapse of disease. This relapse is often caused by an acquired or inherent resistance to the therapy, but in some cases it can also be due to the late detection of recurrence in patients undergoing treatment. Measured at baseline (prior to treatment), serum LDH levels have recently been observed to be a prognostic marker for the OS of patients treated with dabrafenib and trametinib [14]. This study showed that trial patients with lower disease burden $(\mathrm{n}=237)$ had a lower serum LDH and an improved OS (75\% CI: 70-81) measured at 2 years compared with patients with at least two-times the upper limit of normal (7\% OS [3-19\%]) [14]. Since our miRNA panel was found to be superior at determining OS in treatmentnaive patients [9], further investigation into our panel's clinical utility is currently being assessed.

Diagnostic imaging (e.g., CT scan, PET, and MRI) is the mainstay to record baseline images to observe the whereabouts of metastatic deposits. Appropriate therapy is then commenced with 'target' and 'non-target' lesions assessed at intervals of 2-3 months based upon the Response Evaluation Criteria in Solid Tumors version 1.1 (RECIST vl.1) or the immune-related response criteria (irRC) guidelines $[15,16]$. These scans are assessed by the radiologist and the patients are categorized as having a complete response (CR), a partial response (PR), progressive disease (PD) or stable disease (SD). This type of monitoring is limited as it can only be used intermittently during the course of treatment due to the risks of increased radiation exposure (e.g., a CT scans radiation is $\sim 60 \times$ greater than a chest $\mathrm{x}$-ray) [17]. The various imaging techniques also have limits of detection. For example, lesions can only be accurately measured if they are $>10 \mathrm{~mm}$ [15] 
and FDG-PET scans can only detect metabolic activity [18]. In sum, this means that if the lesions are $<10 \mathrm{~mm}$ or they have low metabolic activity, then tumours may not be detected. Lastly, no scans are sensitive enough to detect micrometastases [19]. To address these limitations in monitoring of patients under therapy, we are currently assessing the utility of the MELmiR-7 panel, in comparison with diagnostic imaging, using serially collected bloods. Additionally, in a head-to-head comparison, circulating tumor DNA will also be compared.

Armed with these improved surveillance methodologies, we hope to see better clinical management of disease, which in turn could increase objective clinical responses and survival times. Future melanoma follow-up and treatment regimens should consider the utility of miRNAs as a companion diagnostic and prognostic aid in a clinical setting.

Financial \& competing interests disclosure

MS Stark is the named inventor of the miRNA panel discussed in this Editorial. The author has no other relevant affiliations or financial involvement with any organization or entity with a financial interest in or financial conflict with the subject matter or materials discussed in the manuscript apart from those disclosed.

No writing assistance was utilized in the production of this manuscript.

\section{Open access}

This work is licensed under the Attribution-NonCommercialNoDerivatives 4.0 Unported License. To view a copy of this license, visit http://creativecommons.org/licenses/ by-nc-nd/4.0/

\section{References}

1 Australian Institute of Health and Welfare (AIHW).

www.aihw.gov.au/cancer

2 National Cancer Institute (NIH). https://seer.cancer.gov/archive

3 Ferlay J, Steliarova-Foucher E, Lortet-Tieulent $\mathrm{J}$ et al. Cancer incidence and mortality patterns in Europe: estimates for 40 countries in 2012. Eur. J. Cancer 49(6), 1374-1403 (2013).

4 Balch CM, Gershenwald JE, Soong SJ et al. Final version of 2009 AJCC melanoma staging and classification. J. Clin. Oncol. 27(36), 6199-6206 (2009).

5 Brochez L, Naeyaert JM. Serological markers for melanoma. Br. J. Dermatol. 143(2), 256-268 (2000).

6 Finck SJ, Giuliano AE, Morton DL. LDH and melanoma. Cancer 51(5), 840-843 (1983).

7 Karakousis CP, Balch CM, Urist MM, Ross MM, Smith TJ, Bartolucci AA. Local recurrence in malignant melanoma: long-term results of the multi-institutional randomized surgical trial. Ann. Surg. Oncol. 3(5), 446-452 (1996).

8 Sirott MN, Bajorin DF, Wong GY et al. Prognostic factors in patients with metastatic malignant melanoma. A multivariate analysis. Cancer 72(10), 3091-3098 (1993).

9 Stark MS, Klein K, Weide B et al. The prognostic and predictive value of melanomarelated microRNAs using tissue and serum: a microRNA expression analysis. EBioMedicine 2(7), 671-680 (2015).

10 Bonazzi VF, Stark MS, Hayward NK. MicroRNA regulation of melanoma progression. Melanoma Res. 22(2), 101-113 (2012).

11 Hodi FS, O’Day SJ, Mcdermott DF et al. Improved survival with ipilimumab in patients with metastatic melanoma. N. Engl. J. Med. 363(8), 711-723 (2010).

12 Sosman JA, Kim KB, Schuchter L et al. Survival in BRAF V600-mutant advanced melanoma treated with vemurafenib. $N$. Engl. J. Med. 366(8), 707-714 (2012).

13 Long GV, Weber JS, Infante JR et al. Overall survival and durable responses in patients with BRAF V600-mutant metastatic melanoma receiving dabrafenib combined with trametinib. J. Clin. Oncol. 34(8), 871-878 (2016).

14 Long GV, Grob JJ, Nathan P et al. Factors predictive of response, disease progression, and overall survival after dabrafenib and trametinib combination treatment: a pooled analysis of individual patient data from randomised trials. Lancet Oncol. 17(12), 1743-1754 (2016).

15 Eisenhauer EA, Therasse P, Bogaerts J et al. New response evaluation criteria in solid tumours: revised RECIST guideline (version 1.1). Eur. J. Cancer 45(2), 228-247 (2009).

16 Hodi FS, Hwu WJ, Kefford R et al. Evaluation of immune-related response criteria and RECIST v1.1 in patients with advanced melanoma treated with pembrolizumab. J. Clin. Oncol. 34(13), 1510-1517 (2016).

17 Brenner DJ, Hall EJ. Computed tomographyan increasing source of radiation exposure. N. Engl. J. Med. 357(22), 2277-2284 (2007).

18 Kong BY, Menzies AM, Saunders CA et al. Residual FDG-PET metabolic activity in metastatic melanoma patients with prolonged response to anti-PD-1 therapy. Pigment Cell Melanoma Res. 29(5), 572-577 (2016).

19 Kumar R, Alavi A. Clinical applications of fluorodeoxyglucose-positron emission tomography in the management of malignant melanoma. Curr. Opin. Oncol. 17(2), 154-159 (2005). 